\title{
A study of leukemias Profile in central India
}

\section{Original Research Article}

\author{
Ahirwar R. ${ }^{1}$, Nigam R.K. ${ }^{2}$, Parmar D. ${ }^{3}$ \\ ${ }^{1}$ Dr. Rajnikant Ahirwar, Assistant Professor, Department of Pathology, RKDF Medical College, Bhopal, ${ }^{2}$ Dr. Rajendra \\ Kumar Nigam, Professor, Department of Pathology, Gandhi Medical College, Bhopal, ${ }^{3}$ Dr. Deepankar Parmar, Professor, \\ Department of Pathology, RKDF Medical College, Bhopal, MP, India.
}

Corresponding Author: Dr. Rajnikant Ahirwar, A-2 Apartment, Flat No-301, Rchilifescape, Jatkhedi, Bhopal. E-mail: drrajnikantahirwar@gmail.com

\begin{abstract}
Introduction: Leukemia was considered as a rare disease few years back. However, it is increasing in incidence and prevalence slowly and steadily. Leukemias are euplastic proliferations of haematopoietic cells and form a major proportion of haematopoietic neoplasms that are diagnosed worldwide. Objective: To find out the incidence of four major types of leukemias in central India. Methods: Total 73 cases were selected from in and out patient departments of Gandhi Medical College and associated Hamidia Hospital Bhopal over a period from Oct 2013 to Nov 2014. Diagnosis was based on peripheral blood count, peripheral blood smear and bone marrow examination for morphology along with cytochemistry study whenever required. Results: Out of 73 cases on final diagnosis, 23 cases $(31.51 \%)$ were of Acute Lymphoblastic Leukemias, 11 cases (15.07\%) were of Acute Myeloblastic Leukemias, 35 cases (47.97\%) were of Chronic Myeloid Leukemias, 1 case $(1.37 \%)$ is of Chronic Lymphocytic Leukemia and 3 cases (4.11\%) were Undiagnosed which were referred to higher centers. Conclusion: The present study revealed that Chronic leukemia was more common than acute leukemia with Chronic Myeloid Leukemias being the most common type, followed by Acute Lymphoblastic Leukemias, Acute Myeloblastic Leukemias and Chronic Lymphocytic Leukemia.
\end{abstract}

Keywords: Acute Lymphoblastic Leukemias, Incidence, Leukaemia.

\section{Introduction}

Leukemia was considered as a rare disease few years back. However, it is increasing in incidence and prevalence slowly and steadily. Leukemias are the 10th most common cancer in men and $12^{\text {th }}$ most common in women and constitute $3 \%$ of the global cancer burden [1].

Developing countries bear more than half of global cancer burden, because $75 \%$ of the world population lives in these countries [2]. The incidence of Leukemia is highest in North America and Australia/New-Zealand and lowest in sub-Saharan Africa [3].

In India,lympho-haematopoietic malignancies constitute $9.5 \%$ of all cancers in men and $5.5 \%$ in women [4].

Manuscript received: $4^{\text {th }}$ April 2018

Reviewed: $14^{\text {th }}$ April 2018

Author Corrected: $20^{\text {th }}$ April 2018

Accepted for Publication: $25^{\text {th }}$ April 2018
As per available information from population-based surveys, the incidence of leukemia in India varies from $0.8 / 1,00,000$ in Barshi (Rural area of Maharashtra) to $5 / 1,00,000$ in Delhi.

These figures are comparably lower than rest of the world but under diagnosis and under-reporting cannot be ruled out [4]. The cell type distribution of leukemias observed in India is different from that observed in developed world.

Myeloid leukemias predominate in India while lymphoid leukemias dominate in western world mainly because of higher incidence of chronic lymphatic leukemia [5].

Despite being relative uncommon, leukemias have been studied more extensively because of easy accessibility of involved tissue [1]. 


\section{Original Research Article}

The incidence of CML was noted highest (45.3\%) and that was lowest of CLL (5.7\%) in Capital of India i.e. Delhi during a period of 1970-1979.19 Similar observations were noted in Chandigarh and other metro cities like Mumbai and Calcutta [6,7,8].

There was exception for incidence of ALL (39.2\%) which was highest observed in Kerala state during a period of 1980-1983 [9]. The leukemias are defined as diseases in which abnormal proliferation of haematopoetic cells cause progressively increasing infiltration of bone marrow, although in certain forms the lymphatic tissues are particularly affected [10].

Malignant proliferation of haematopoietic cells (leukemia) constitutes major proportion of haematopoietic neoplasm's worldwide. Leukemias are classified into myeloid and lymphoid subtype [11].

Typing of leukemia is essential for effective therapy because prognosis and survival rate are different for each type and sub-type [12]. Leukemia are of two types; acute and chronic.

Acute leukemias are; acute lymphoblastic leukemia (ALL) and acute myeloid leukemia (AML). In childhood, ALL is most common type than AML. In India, the incidence of ALL and AML are $35 \%$ and $15 \%$ of all hematological malignancies respectively.

Chronic leukemias are classified into chronic myeloid leukemia (CML) and chronic lymphocytic leukemia (CLL) $[13,14]$. Due to the lack of any nationwide leukemia screening program, most of the population of India is still unaware of this blood disorder.

Lack of awareness also plays a role in underlying late presentation and noncompliance with screening guidelines [15].

Hence it is important to determine the current incidence of leukemia's in India as well as to understand how the occurrence and outcome of the disease differs across the whole country.

\section{Materials and Methods}

\section{Results}

The present study comprises 73 cases of Leukemia. The observations made in this study are as follows.
Place of study- Department of Pathology, Gandhi Medical College, Bhopal

Type of study- prospective

Sampling methods- Selected from in and out patient departments of Gandhi Medical College and associated Hamidia

Sample collection- Blood, bone marrow smear

Inclusion criteria- having provisional diagnosis of acute leukemia and leucoproliferative disorder.

The study was conducted after approval from institutional ethical committee in the Department of Pathology, Gandhi Medical College, Bhopal from Oct 2013 to Nov 2014.

Total 73 cases were selected from in and out patient departments of Gandhi Medical College and associated Hamidia Hospital Bhopal having provisional diagnosis of acute leukemia and leucoproliferative disorder so on basis of leishman stained smears-Peripheral/bone marrow along with cytochemistry study whenever required.

Thorough case history and clinical examination as per predefined Performa with Hemoglobin estimation, WBC count, Platelet count and various hematological indices were evaluated using automated analyzer.

Peripheral blood/bone marrow smear was made on clean glass slides with fresh blood samples; smears were fixed and stained by leishman stain.

Leishman stain was poured on air dried unfixed smears for a period of 5-7 minutes, then added twice quantity of buffered distilled water for next 10 minutes. Smears prepared on clean glass slides, fixed by methanol and stained for various cytochemical stains (MPO, PAS, NES, and NAP).

For all the special stains, commercially available kits (Leucognost) were used. FAB classification of acute leukemia was applied for sub-typing. 
Table No-1: Type of Leukemia's, including Age, sex distribution.

\section{Original Research Article}

\begin{tabular}{|c|c|c|c|c|c|c|c|c|c|c|}
\hline \multirow{3}{*}{$\begin{array}{c}\text { Age (yrs) } \\
\text { \& Sex } \\
0-15\end{array}$} & \multicolumn{10}{|c|}{ No of Patient } \\
\hline & \multicolumn{2}{|c|}{ ALL (23) } & \multicolumn{2}{|c|}{ AML (11) } & \multicolumn{2}{|c|}{ CML (35) } & \multicolumn{2}{|c|}{ CLL (1) } & \multicolumn{2}{|c|}{ Undiagnosed (3) } \\
\hline & 13 & $56.52 \%$ & 2 & $18.18 \%$ & 3 & $8.57 \%$ & 0 & 0 & 0 & 0 \\
\hline $16-30$ & 5 & $21.74 \%$ & 4 & $36.36 \%$ & 9 & $25.71 \%$ & 0 & 0 & 2 & $67.00 \%$ \\
\hline $31-45$ & 3 & $13.04 \%$ & 4 & $36.36 \%$ & 13 & $37.14 \%$ & 0 & 0 & 1 & $33.00 \%$ \\
\hline$>45$ & 2 & $8.70 \%$ & 1 & $9.09 \%$ & 10 & $28.57 \%$ & 1 & $100 \%$ & 0 & 0 \\
\hline Male & 15 & $65.22 \%$ & 4 & $36.36 \%$ & 10 & $28.57 \%$ & 0 & 0 & 2 & $67.00 \%$ \\
\hline Female & 8 & $34.78 \%$ & 7 & $63.64 \%$ & 25 & $71.43 \%$ & 1 & $100 \%$ & 1 & $33.00 \%$ \\
\hline
\end{tabular}

Out of 73 cases on final diagnosis, 23 cases (31.51\%) were of Acute Lymphoblastic Leukemias, 11 cases (15.07\%) were of Acute Myeloblastic Leukemias, 35 cases (47.97\%) were of Chronic Myeloid Leukemias, 1 case (1.37\%) is of Chronic Lymphocytic Leukemia and 3 cases $(4.11 \%)$ were Undiagnosed.

In Our study Out of 73 cases - 31cases (42.47\%) were Male and 42cases (57\%) were Female. which shows female predominance it may be due to sample size. Out of 73 cases -18 cases (24.66\%) were of age group 0 to 15 years, 21 cases $(28.77 \%)$ were of age group 16 to 30 years, 19 cases (26.03\%) were of age group 31 to 45 years and 15 cases (20.55\%) were of above 45 years.

Hemoglobin Distribution: In Acute Lymphoblastic Leukemia and Acute Myeloblastic Leukemia most of the cases (56.52\% and (72.73\%) had less than $6 \mathrm{gm} \% \mathrm{Hb}$. In Chronic Myeloid Leukemia only 5.71\% cases had severe anemia, while $34.29 \%$ and $51.43 \%$ had moderate and mild anemia. In Chronic Lymphocytic Leukemia patient had mild anemia.

Total Leukocyte Count Distribution: In Acute Lymphoblastic Leukemia and Acute Myeloblastic Leukemia 39.13\% and 63.64\% of cases showed countless then 4000/cu mm. While in Chronic Myeloid Leukemia most of cases (91.43\%) showed leukocytosis and $100.00 \%$ cases of Chronic lypmphocytic leukemia showed leukocytosis.

Platelet Count Distribution: Most of the cases (82.61\% and 100\%) of acute lymphoblastic leukemia (ALL) acute myeloid leukemia (AML) had moderate to severe thrombocytopenia. Whereas in chronic myeloid leukemia (CML) and chronic lypmphocytic leukemia (CLL) most of cases had platelet above 1lakh/cu mm.

Sub-classification Acute Myeloblastic Leukemia: On by morphological and cytochemical method. sub classification of Acute Myeloblastic Leukemia is done. Most common subtype of Acute Myeloblastic Leukemia was M3 subtype which comprised of $36.36 \%$ cases.

Whereas M1, M2, M5, M6, M7 comprised of $18.18 \%, 9.09 \%, 9.09 \%, 9.09 \%, 18.18 \%$ respectively. In present study there were no cases belonging to M0 and M4 subtype. 
Table-2: Frequency (in percentage) of various Leukemias in India.

\section{Original Research Article}

\begin{tabular}{|c|c|c|c|c|c|c|}
\hline Reference & Region (Period of Study) & No of cases & ALL & AML & CML & CLL \\
\hline Chatterjee et al & Calcutta (1949-1961) & 544 & 22.5 & 32.5 & 35.9 & 5.9 \\
\hline Advani et al & Mumbai (1960-1975) & 1126 & 30 & 13 & 40 & 9 \\
\hline Prakash et al & Pondicherry (1970-1979) & 278 & 35 & 29.5 & 30.8 & 3.2 \\
\hline Rani et al & Delhi (1970-1979) & 490 & 15.5 & 30.8 & 45.3 & 5.7 \\
\hline Verghese et al & Kerala (1980-1983) & 1016 & 39.2 & 19.6 & 16.4 & 1.9 \\
\hline Kushawaha et al & Lucknow (1971-1984) & 970 & 9.3 & 38.7 & 48 & 2.6 \\
\hline Shome et al & Chandigarh (1975-1983) & 820 & 24 & 29.3 & 36.7 & 8.8 \\
\hline Dicosta et al & Mumbai (1975-1984) & 242 & 36 & 22 & 38 & 2 \\
\hline Rathee et al & Haryana (2008-2012) & 650 & 17.2 & 33.8 & 39 & 10 \\
\hline Jaya Bhaskar & Loni, Maharashtra & 156 & 26.28 & 23.07 & 33.97 & 15.38 \\
& (2006 and 2011) & & & & & \\
\hline Our study & Bhopal (2013-2014) & 73 & 31.51 & 15.07 & 47.97 & 1.37 \\
\hline
\end{tabular}

\section{Discussion}

The incidence of leukemia has increased considerably and this rise is noticeable because of improved statistics, better case findings with novel technologies which lead to better diagnosis and treatment methods.

This incidence varies in different geographical regions according to varying life styles, economic conditions, and poverty rate [16].

In India the incidence of various hematological cancers is different as compared to western countries. This can be attributable to less health awareness and poor availability of health care delivery system in India [17, 18]. In present study 73 cases of leukemias were studied 34 cases $(46.58 \%)$ and 36 cases $(49.32 \%)$ were of acute and chronic leukemias respectively and 3 cases $(4.11 \%)$ were undiagnosed.

Out of 73 cases - 18 cases $(24.66 \%)$ were of age group 0 to 15 years, 21 cases $(28.77 \%)$ were of age group 16 to 30 years, 19 cases $(26.03 \%)$ were of age group 31 to 45 years and 15 cases $(20.55 \%)$ were of above 45 years. In Acute Lymphoblastic Leukemia and Acute Myeloblastic Leukemia most of the cases $(56.52 \%$ and (72.73\%) had less than 6 gm\% Hb. In Chronic Myeloid Leukemia only $5.71 \%$ cases had severe anemia, while $34.29 \%$ and $51.43 \%$ had moderate and mild anemia.
In Chronic Lymphocytic Leukemia patient had mild anemia In Acute Lymphoblastic Leukemia and Acute Myelo-blastic Leukemia $39.13 \%$ and $63.64 \%$ of cases showed count less then 4000 /cu mm. While in Chronic Myeloid Leukemia most of cases (91.43\%) showed leukocytosis and $100.00 \%$ cases of chronic lypmphocytic leukemia showed leukocytosis.

Most of the cases $(82.61 \%$ and $100 \%)$ of acute lymphoblastic leukemia (ALL) acute myeloid leukemia (AML) had moderate to severe thrombocytopenia. Whereas in chronic myeloid leukemia (CML) and chronic lypmphocytic leukemia (CLL) most of cases had platelet above 1lakh/cu mm.

In present study four special staining procedures were done after making a provisional diagnosis of leukemias by leishman stain (Stain used Myeloperoxidase, Periodic acid Schiff, Non-specific Esterase and alkaline phosphates). On special staining we found Myeloblasts of AML and CML showed positive (coarse brown black granules) staining with MPO in more than $3 \%$ of blasts in all cases except one in which NSE stain was positive.

Whereas ALL and CLL were negative for MPO stain. The lymphoblasts of ALL and CLL showed positive (block positive) PAS staining. 


\section{Original Research Article}

Whereas myeloblasts of AML and CML were negative. The NAP scoring was done in all (35) cases of CML and the range of NAP score was 0 to 10 . Undiagnosed 3 cases were negative for all (MPO, PAS, NSE) stain. They were advised for flow cytometry, immunophenotyping for which they referred to higher center.

Among all leukemias the incidence and prevalence of chronic myeloid leukemia (CML) remains higher due to chronicity, earlier diagnosis because of accurate and cheaper diagnostic tool and greater physician awareness.

The studies done by various authors also observed [6,8,19-23] higher incidence of chronic myeloid leukemia (CML) than ours while the findings of Menzes and Malik [24] and Verghese et al [9]vary from the above studies (Table 2).

Our study revealed chronic myeloid leukemia (CML) $(47.95 \%)$ as the most common type of leukemia (Table 1). The varying findings of Menzes and Malik [24] and Verghese et al [9] can be attributable to geographical variation and population bias.

The incidence of acute lymphoblastic leukemia was higher in studies by Prakash et al [25] and Verghese et al [9]. In our study the cases of acute lymphoblastic leukemia were the second predominant $(31.51 \%)$ which correlates with study of Advani et al [26], Dicosta et al [7], Modak H et al [27], Chen et al [28] and Shome et al [6] (Table 1).

The incidence of Acute myeloid leukemia (AML) in our study was the third most common. Similar findings are reported by Advani et al [26] and D'Costa GG et al [7]. Incidence of higher percentage than ours was reported by Chatterjee et al [8], Prakash et al [25], Rani et al [21], Kushwaha et al [20], Shome et al [6], and Rathee et al [23] (Table 8).

Our study shows the incidence of chronic lypmphocytic leukemia (CLL) to be lowest (1.37\%) among all the four major types of leukemia which correlates well with the findings of Kushwaha et al [20], Verghese et al [9] and D'Costa GG et al [7] (Table 2).

It has been seen that the spectrum of cancer epidemiology seen in India is different than that seen in any developed country. It should be stressed that there are not many cancer registry data's in India despite a large population, so better development of regional and national registries is the need of the hour.

\section{Conclusion}

Present study concluded that the incidence of different types of leukaemia in central India doesn't differ markedly from rest of the Indian populations. Chronic leukemia was more common than acute leukemia with chronic myeloid leukemia being the most common type, followed by Acute Lymphoblastic Leukemias, Acute Myeloblastic Leukemias and Chronic Lymphocytic Leukemia.

Chronic myeloid leukemia which came out to be more common in our study, is mainly a leukemia of adults affecting the myeloid series while AML occurring in younger age group is characterized by presence of $>20 \%$ blasts in marrow, as per the WHO criteria.

This incidence varies in different geographical regions according to varying life styles, economic conditions, and poverty rate. It should be stressed that there are not many cancer registry data's in India despite a large population, so better development of regional and national registries is the need of the time.

Addition to existing knowledge: Though numerous studies of leukemia have been done in various regions of India but this study is exclusively the first one which details the leukemia profile in central part of India.

Funding: Nil, Conflict of interest: None initiated Permission from IRB: Yes

\section{References}

1. Parkin DM, Pisani P, Ferlay J. Estimates of the world wide incidence of eighteen major cancers in 1985. Int J Cancer. 1993 Jun 19;54(4):594-606.

2. WHO 1999. Health situation in the South East Asia Region 1994-1997, Regional Office for SEAR, New Delhi, 1999.

3. Parkin DM, Whelam SL, Ferlay J, Raymond L, Young J, eds., Cancer incidence in five continents, Vol VII IARC Scientific publications number 143 Lyon 1997, International agency for research on cancer pp 804-913. 


\section{Original Research Article}

4. National cancer Registry programme, consolidated report of population based cancer registries 1990-96. Incidence and distribution of cancer Indian council of Medical Research, New Delhi, Aug 2001.

5. Prakash S, Ramamurthi, Gopalan R, Aurora AL. Leukaemias at Pondicherry. Indian J Cancer. 1981 Mar; 18 (1):1-6.

6. Shome DK, Ghosh K, Mohanty D, Das KC. Leukaemia in north-west India. Acta Haematol. 1985; 73 (4):244.

7. D'Costa G, Siddiqui HM, Pradhan RM, Gupte SS. Pattern of leukemias: a ten-year incidence study of 242 cases. J Postgrad Med. 1989 Oct;35(4):191-5.

8. B, Ghose S, Ray RN. Incidence of leukaemia. (An analysis of 544 cases studied in Calcutta). J Assoc Physicians India. 1962 Dec;10:673-6.

9. Varghese PR, Elayidom NB, Joseph CD, Kumar S. Epidemiological observations on leukaemia in Kerala (A study of 1016 cases over three years), Ind J Haematol, 1984;2:15-17.

10. Robbins and Cotran: Pathologic Basis of Diseases, 7th Edition. Elsevier Publications, 1999. Pg. 232-37.

11. Harris NL, Jaffe ES, Vardiman JW, Stein H, Diabold J, Flandrin G. WHO Classification of tumors of haematopoietic and lymphoid tissues- Introduction. In: Harris NL, Jaffe ES, Vardiman JW, editors. Pathology and genetics of tumors of haematopoietic and lymphoid tissues. Lyon France: IARC press; 2008: p 1-15.

12. Salkar AB, Patrikar A, Bothale K, Malore S, Salkar A, Modani S. Clinicohematological evaluation of leukemias in a tertiary care hospital. IOSR-JDMS. 2014; 13:126-34

13. McKenna RW. Multifaceted approach to the diagnosis and classification of acute leukemias. Clin Chem. 2000 Aug;46(8 Pt 2):1252-9.

14. Arber DA, Cousar J. Hematopoeitic Tumors: Principles of pathologic diagnosis. In: Greer JP, Rodgers GM, Foerster J, Paraskevas F, Lukens JN, Glader B, editors. Wintrobe's Clinical Hematology. 13th edition, vol 2. Philadelphia: Lipincotts Williams and Wilkins; 2014: p 1663- 68.

15. Radha RatheemI NAKSHI Vashisht, Ashok Kumar, Sunita Singh. Incidence of acute and chronic forms of leukemia In Haryana. Int. $\mathbf{J}$ of Pharmacy and Pharmaceutical Sciences. 2014;6(2):323-325.

16. Gunz FW. The epidemiology and genetics of the chronic leukaemias.Clin Haematol. 1977 Feb;6(1):3-20.

17. Kumar L, Kumari M, Kumar S, Kochupillai V, Singh R, Clinical and laboratory features at diagnosis in 437 patients with chronic myelogenous leukemia: An experience at tertiary care centre, In: Kumar L (ed). Progress in hematologic oncology, New York: The advanced research foundation 2003;83-98.

18. Kulshrestha R, Sah SP. Pattern of occurrence of leukemia at a teaching hospital in eastern region of Nepal - a six year study. JNMA J Nepal Med Assoc. 2009 Jan-Mar;48(173):35-40.

19. Vasavada PS, Akbar Ali and DP Mukherjee Clinical studies in leukemia- A review of 100 cases in symposium in leukemia. Leukemia journal of associations Phy India, 1962;9:357.

20. Kushwaha MR, Bagchi M, Mehrotra RM. Leukaemias at Lucknow--a study of 200 cases. Indian J Cancer. 1978 Sep;15(3):28-34.

21. Rani S, Beohar PC, Mohanty TK, Mathur MD. Leukaemic pattern in Delhi--a ten year study of 490 cases. Indian J Cancer. 1982 May-Jun;19(2):81-6.

22. Jaya Bhaskar Baviskar, Incidence of acute and chronic leukemias in rural area at tertiary care teaching hospital: a five years of study, Indian Journal of Pathology and Oncology, October-December 2016;3(4); 710-713.

23. Radha Rathee, Minakshi Vashist, Ashok Kumar, Sunita Singh, Incidence of acute and chronic forms of leukemia in Haryana; International Journal of Pharmacy and Pharmaceutical Sciences; 2014;6(2):42-45.

24. Menezes S, Malik GB. A study of leukaemia in Goa. Indian J Pathol Microbiol. 1977 Apr; 20 (2): 83-90. 


\section{Original Research Article}

25. Prakash S, Ramamurthi, Gopalan R, Aurora AL. Leukaemias at Pondicherry. Indian J Cancer. 1981 Mar; 18 (1):1-6.

26. Advani SH,Jussawala DJ,Nagaraj RD, Gangadharan P, Shetty PA. A study of 1226 leukemia casesEpidemiologic and end result analysis. The Indian $\mathbf{J}$ of Cancer, 1979; 16: 8-17
27. Modak H, Kulkarni SS, Kadakol GS, Hiremath SV, Patil BR, Hallikeri U, Gai PB. Prevalence and risk of leukemia in the multi-ethnic population of North Karnataka. Asian Pac J Cancer Prev. 2011;12(3):671-5.

28. Chen B., Huang Z., Zhang X., Ou-Yang J., Li J., Zhai $Y$. et al. An epidemiological investigation of leukemia incidence between 2003 and 2007 in Nanjing, China. J Hematol Oncol 2010;3:21.

\section{How to cite this article?}

Ahirwar R, Nigam R.K, Parmar D. A study of leukemias Profile in central India.Trop J Path Micro 2018;4(2):181-187. doi: 10.17511/jopm.2018.i2.12 